COMMUNICATIONS IN

ANALYSIS AND GEOMETRY

Volume 14, Number 3, 551-564, 2006

\title{
A New Li-Yau-Hamilton Estimate for the Ricci Flow
}

\author{
Hsiao-Bing Cheng
}

\section{Introduction.}

Since its discovery, Hamilton's Li-Yau-Hamilton (LYH) estimate has proven its importance in many different works (for example [7], [9], [10], [6]), and as a result, similar inequalities have subsequently appeared in the study of numerous other geometric flows - the mean curvature flow [11], the KählerRicci flow [1], and the Gauss curvature flow [2], among others.

The importance of LYH-type estimates is underscored by the fact that the discovery of an LYH estimate without curvature assumption is a large step in Hamilton's program for Geometrization. Traditionally, positivity of curvature in some form has always been needed for the existence of an LYH estimate. However, work of Hamilton [10] and Ivey [13] in three dimensions indicate that because the curvature operator becomes (in a sense) close to positive near singularities, and because there is an LYH estimate for positive curvature operator, there should be an LYH estimate without any curvature assumptions. Because of this, one approach towards finding an inequality on spaces of arbitrary curvature is to perturb the LYH estimates that are found when there is positive curvature. In fact, using this point of view, an LYH estimate was discovered on surfaces where some negative curvature was allowed [12]. This provides great motivation to find and understand the LYH estimates that do exist when positivity is assumed, and to discover the deeper reasons why such inequalities exist.

One very interesting approach towards understanding LYH estimates attempts to view these inequalities in a geometric setting. This was first accomplished in the work of Chow and $\mathrm{Chu}$ [3], where a degenerate metric and a space-time connection is place on the flow. In this setting, Hamilton's original LYH quantity appears very naturally and geometrically, being closely related to the curvature of this conenction. In subsequent papers, Chow and Chu [4], as well as Chow and Knopf [5] have since gained more understanding of this point of view. They have been able to refine it, clarify- 
ing the correspondence between Hamilton's LYH quantity and the curvature of the chosen connection. In fact, many of the ideas and expressions found in Hamilton's original paper appear quite naturally in terms of the geometry of the chosen metric and connection. Moreover, by examining different space-time connections, new and different LYH estimates are discovered.

We also note that though Perelman's recent work on the Geometrization conjecture [14] does not involve LYH estimates with no sign assumption, it does use ideas inspired from the space-time point of view.

Here, we generalize Chow and Knopf's work to obtain a new LYH estimate for the Ricci flow. We prove the following Theorem.

Theorem 1.1. Let $\frac{\partial}{\partial t} g=-2 R c$. Let $A$ be a closed 2-form solving

$$
\frac{\partial}{\partial t} A=\Delta_{d} A
$$

and $h$ a symmetric 2-tensor solving

$$
\frac{\partial}{\partial t} h=\Delta_{L} h+\nabla \nabla|A|^{2} .
$$

Then, the inequality

$$
\Phi=R_{i j k l} U_{i j} U_{l k}+2 W_{j} D_{j} A_{k l} U_{l k}+\left(g^{p q} A_{j p} A_{l q}+h_{j l}\right) W_{j} W_{l} \geq 0,
$$

is preserved for $t>0$. Here, $\Delta_{d}$ is the Hodge-deRham Laplacian on twoforms, and $\Delta_{L}$ is the Lichnerowicz Laplacian on symmetric tensors.

In the first three parts of this paper, we discuss Hamilton's original estimate, the space-time viewpoint of Chow and $\mathrm{Chu}$, and then the new expression discovered by Chow and Knopf. We then state our generalization of Chow and Knopf's theorem; discuss briefly the orthonormal frame bundle - which aids us greatly in keeping computations clean - as well as noting the flows of some of our important quantities. Finally, we prove our theorem.

\section{Hamilton's Original Estimate.}

In 1991, Hamilton discovered a differential Harnack estimate for the Ricci flow. His theorem states that:

Theorem 2.1. If $\left(M^{n}, g\right)$ has weakly positive curvature operator, then for any two-form $U_{i j}$ and any one-form $W_{i}$, we have

$$
M_{i j} W_{i} W_{j}+2 P_{i j k} U_{i j} W_{k}+R_{i j k l} U_{i j} U_{l k} \geq 0
$$


where

$$
M_{i j}=\Delta R_{i j}-\frac{1}{2} D_{i} D_{j} R+2 R_{i k l j} R_{k l}-R_{i k} R_{j k}+\frac{1}{2 t} R_{i j}
$$

and

$$
P_{i j k}=D_{i} R_{j k}-D_{j} R_{i k}
$$

$B y$ weakly positive curvature operator, we mean that for any two-form $U$, the curvature tensor satisfies

$$
R_{i j k l} U_{i j} U_{l k} \geq 0 .
$$

This condition is preserved under the Ricci flow.

Remark 2.2. In dimension three, weakly positive curvature operator is equivalent to weakly positive sectional curvature.

Remark 2.3. Here, we are using the convention $g_{m l} R_{i j k}^{m}=R_{i j k l}$ whereas in Hamilton's original paper [8], $g_{m l} R_{i j k}^{m}=R_{i j l k}$.

Remark 2.4. We call this estimate a Li-Yau-Hamilton (LYH) estimate. The quantity

$$
M_{i j} W_{i} W_{j}+2 P_{i j k} U_{i j} W_{k}+R_{i j k l} U_{i j} U_{l k},
$$

we call a $L i-Y a u-H a m i l t o n(L Y H)$ quantity.

Proof. We give a brief description of the proof. The main idea is to compute the evolution equation of the LYH quantity. We assume we are at the first time where there is a point $x \in M$, a two-form $U$, and a one-form $W$ such that

$$
M_{i j} W_{i} W_{j}+2 P_{i j k} U_{i j} W_{k}+R_{i j k l} U_{i j} U_{l k}=0 .
$$

The evolution equation of this quantity then computed. Careful examination of this evolution equation shows it to be essentially the sum of two squares. The maximum principle then prevents the quantity from ever becoming negative.

We note that in the LYH expression, no derivatives of $U$ or $W$ appear. However, the evolution equation of the LYH expression does contain derivatives of $U$ and $W$ in both space and time. Thus, values for these derivatives may be prescribed, making the evolution equation of the LYH expression more manageable. We will use this same idea in the proof of the main theorem.

This LYH quantity was originally derived by considering the behavior of certain quantities on translating Ricci solitons - steady state solutions of the 
Ricci flow which move via diffeomorphisms. In fact, for the correct choice of $U$ and $W$, the LYH quantity (without the time term in $M$ ) is identically zero on a translating soliton. However, there is another way to view the LYH quantity.

\section{Space-Time Formulation.}

In 1995, Chow and Chu [3] discovered a new way of looking at the LYH quantity

$$
Z=M_{i j}^{0} W_{i} W_{j}-2 P_{i j k} U_{i j} W_{k}+R_{i j k l} U_{i j} U_{l k}
$$

where $M_{i j}^{0}$ is just $M_{i j}$ without the time term:

$$
M_{i j}^{0}=\Delta R_{i j}-\frac{1}{2} D_{i} D_{j} R+2 R_{i k l j} R_{k l}-R_{i k} R_{j k} .
$$

Hamilton's proof of the LYH estimate works to show that if $Z$ is weakly positive for any $U$ and $W$ initially, then it remains so. We use this expression instead of the full LYH expression to make this exposition more simple.

Remark 3.1. In this, as well as the next section, we will be working with a degenerate metric, so we will be more careful about raised and lowered indices when necessary.

Here, we consider a degenerate metric $\tilde{g}^{\alpha \beta}$ on the space-time $\tilde{M}=M^{n} \times$ $[0, T)$ defined by

$$
\tilde{g}^{\alpha \beta}= \begin{cases}g^{i j} & \text { if } 1 \leq \alpha, \beta \leq n \\ 0 & \text { if } \alpha=0 \text { or } \beta=0\end{cases}
$$

For this metric, we can define a connection $\tilde{\nabla}$ by

$$
\begin{aligned}
\tilde{\Gamma}_{i j}^{k} & =\Gamma_{i j}^{k}, \\
\tilde{\Gamma}_{0 j}^{k} & =-R_{j}^{k}, \\
\tilde{\Gamma}_{00}^{k} & =-\frac{1}{2} \nabla^{k} R, \\
\tilde{\Gamma}_{00}^{0} & =\tilde{\Gamma}_{i j}^{0}=\tilde{\Gamma}_{0 j}^{0}=0,
\end{aligned}
$$

where $i, j, k \geq 1$.

Now, the curvature for this connection may be defined in the usual way:

$$
\tilde{R}(X, Y) Z=\tilde{\nabla}_{X} \tilde{\nabla}_{Y} Z-\tilde{\nabla}_{Y} \tilde{\nabla}_{X} Z-\tilde{\nabla}_{[X, Y]} Z
$$


We have all the usual symmetries, and may compute, explicitly, the curvature:

$$
\begin{aligned}
\tilde{R}_{j k l}^{l} & =R_{i j k}^{l}, \\
\tilde{R}_{i j 0}^{l} & =\nabla_{j} R_{i}^{l}-\nabla_{i} R_{j}^{l}, \\
\tilde{R}_{i 0 k}^{l} & =\nabla_{k} R_{i}^{l}-\nabla^{l} R_{i k}, \\
\tilde{R}_{i 00}^{l} & =\Delta R_{i}^{l}-\frac{1}{2} \nabla_{i} \nabla^{l} R+2 g^{l m} R_{p i m}^{q} R_{q}^{p}-R_{m}^{l} R_{i}^{m}, \\
\tilde{R}_{j k l}^{0} & =\tilde{R}_{i 0 k}^{0}=0 .
\end{aligned}
$$

Now, we can lower the indices on our space-time curvature:

$$
\tilde{R}_{\alpha \beta \mu \nu}= \begin{cases}\tilde{R}_{\alpha \beta \mu}^{\gamma} g_{\gamma \nu} & \text { if } \nu \geq 1 \\ -\tilde{R}_{\alpha \beta 0}^{\gamma} g_{\gamma \mu} & \text { if } \nu=0 \text { and } \mu \geq 1 \\ 0 & \text { if } \mu=\nu=0\end{cases}
$$

and then, we see that we have exactly

$$
\begin{aligned}
\tilde{R}_{i j k l} & =R_{i j k l}, \\
\tilde{R}_{0 j k l} & =P_{l j k}, \\
\tilde{R}_{i 00 l} & =M_{i l} .
\end{aligned}
$$

Consider, Rm as a symmetric quadratic form on $\bigwedge^{2} T^{*} \tilde{M}$ with

$$
\tilde{\operatorname{Rm}}(\tilde{S}, \tilde{T})=\sum_{\alpha, \beta, \mu, \nu=0}^{n} \tilde{R}_{\alpha \beta \mu \nu} \tilde{S}^{\alpha \beta} \tilde{T}^{\nu \mu} .
$$

Now, identify $\bigwedge^{2} T^{*} \tilde{M} \cong \bigwedge^{2} T^{*} M \oplus \bigwedge^{1} T^{*} M$ by identifying the space-time tensor $\tilde{T}$ with $U \oplus W$, where $U$ is a two-form and $W$ is a one-form. More precisely, we have

$$
\begin{aligned}
\tilde{T}^{i j} & =U^{i j} \\
\tilde{T}^{0 j} & =-\tilde{T}^{j 0}=W^{j} .
\end{aligned}
$$

Then, the LYH quantity corresponds precisely to the curvature of our spacetime metric:

$$
\tilde{\operatorname{Rm}}(\tilde{T}, \tilde{T})=M_{i j}^{0} W_{i} W_{j}+2 P_{i j k} U_{i j} W_{k}+R_{i j k l} U_{i j} U_{l k} .
$$


Remark 3.2. Our degenerate metric (as well as associated Levi-Civita connection, and curvature) may be thought of as a limit of "stretched" metrics defined by:

$$
\tilde{g}_{N, K}(x, t)=g(x, t)+\left(R+\frac{N}{2(t+K)}\right) d t^{2} .
$$

Let $\tilde{\nabla}_{N, K}$ be the Levi-Civita connection of $\tilde{g}_{N, K}$. When $N \rightarrow \infty$, and then $K \rightarrow \infty$, we obtain $\tilde{\nabla}_{N, K} \rightarrow \tilde{\nabla}$.

In fact, if we use $N \rightarrow \infty$, and $K \rightarrow 0$, we get the full LYH quantity, with the time term:

$$
\tilde{\operatorname{Rm}}_{N, K}(U \oplus W, U \oplus W) \longrightarrow Z+\frac{1}{2 t} R_{i j} W_{i} W_{j}
$$

\section{New LYH Estimate From Space-Time Approach.}

In 2001, B. Chow and D. Knopf [5] used the space-time point of view to derive a new Li-Yau-Hamilton estimate. They consider a very large class of connections on space-time generalizing the one used above. Essentially, they consider adding arbitrary two-forms to the $\Gamma_{0 j}^{k}$ term, one-forms to the $\Gamma_{00}^{k}$ term, and functions to the $\Gamma_{00}^{0}$ term. Certain desirable, as well as natural, conditions are then put on these connections. As in above, the curvatures of these connections (satisfying the desirable conditions) give LYH quantities.

This, leads Chow and Knopf to the following theorem.

Theorem 4.1. If $\left(M^{n}, g\right)$ satisfies the Ricci flow, $A_{0}$ is a closed two-form, $E_{0}$ is a closed one-form, then there exist $A(t)$ and $E(t)$ such that $A(0)=A_{0}$, $E(0)=E_{0}$, and

$$
\frac{\partial}{\partial t} A=\Delta_{d} A
$$

and

$$
\frac{\partial}{\partial t} E=\Delta_{d} E-d|A|_{g}^{2}
$$

where $\Delta_{d}$ is the Hodge Laplacian. Now if

$$
R_{i j k l} U^{i j} U^{l k}+2 \nabla_{j} A_{k l} W^{j} U^{k l}+\left(g^{p q} A_{j p} A_{l q}-\nabla_{j} E_{l}\right) W^{j} W^{l} \geq 0
$$

for all two-forms $U$ and one-forms $W$ at time $t=0$, then it remains positive as long as the Ricci flow exists.

Proof. We briefly describe the proof here. The LYH quantity is essentially the curvature of these space-times. It is shown that the space-time curvature 
satisfies a reaction-diffusion equation. A maximum principle (which must first be shown to hold) is then applied to this equation.

\section{A New Li-Yau-Hamilton Estimate.}

We now generalize Chow and Knopf's LYH estimate. By our conditions on $E$, we have that the two-tensor $\nabla_{i} E_{j}$ is symmetric, and also that its evolution equation is

$$
\frac{\partial}{\partial t} \nabla E=\Delta_{L} \nabla E+\nabla \nabla|A|^{2}
$$

where $\Delta_{L}$ is the Lichnerowicz Laplacian

$$
\Delta_{L} T_{i j}=\Delta T_{i j}+2 R_{k i j l} T_{k l}-R_{i k} T_{j k}-R_{j k} T_{i k} .
$$

In place of $\nabla E$, we now consider a general $(0,2)$-tensor $h . \nabla E$ is symmetric, so we assume $h$ is as well. Similarly, we assume

$$
\frac{\partial}{\partial t} h=\Delta_{L} h+\nabla \nabla|A|^{2}
$$

With such $h$, we obtain the following theorem.

Theorem 5.1. Let $\frac{\partial}{\partial t} g=-2 R c$. Let $A$ be a closed 2-form solving

$$
\frac{\partial}{\partial t} A=\Delta_{d} A
$$

and $h$ a symmetric two-tensor solving

$$
\frac{\partial}{\partial t} h=\Delta_{L} h+\nabla \nabla|A|^{2}
$$

Then, the inequality

$$
\Phi=R_{i j k l} U_{i j} U_{l k}+2 W_{j} D_{j} A_{k l} U_{l k}+\left(g^{p q} A_{j p} A_{l q}+h_{j l}\right) W_{j} W_{l} \geq 0
$$

is preserved. Here, $\Delta_{d}$ is the Hodge-deRham Laplacian, and $\Delta_{L}$ is the Lichnerowicz Laplacian.

Remark 5.2. If we let $h_{i j}$ be $\nabla_{i} E_{j}$ from Chow and Knopf's Theorem, then we recover their Theorem. 


\section{The Orthonormal Frame Bundle.}

To make computations cleaner, we take a point of view that Hamilton uses to prove his LYH estimate.

We consider the frame bundle $F(M)$ of $M$. Points on the space $F(M)$ consist of a point $x$ on $M$, and a frame of vectors $\left\{Y_{1}, Y_{2}, \ldots, Y_{n}\right\}$ on $T_{x} M$. We think of $(k, 0)$-tensors as multi-valued functions on $F(M)$. For example, if $V$ is a covecter, then it takes $\left\{Y_{1}, Y_{2}, \ldots, Y_{n}\right\}$ to the $n$ values $\left\{V\left(Y_{1}\right), V\left(Y_{2}\right), \ldots, V\left(Y_{n}\right)\right\}$. We will abbreviate this by $V=\left\{V_{a}\right\}$, where $V_{a}=V\left(Y_{a}\right)$. Similarly, if $V$ were a $(2,0)$-tensor, it takes $\left\{Y_{1}, Y_{2}, \ldots, Y_{n}\right\}$ to the $n^{2}$ values $\left\{V_{a b}\right\}$, where $V_{a b}=V\left(Y_{a}, Y_{b}\right)$.

Now, covariant differentiation takes a tensor and gives us a new one. Vector fields take functions to functions, so in this setting, we can think of covariant differentiation, $D_{a}$, in the direction $Y_{a}$ as a vector field where if $V=\left\{V_{b}\right\}$, then $D_{a} V$ is a $(2,0)$-tensor $\left\{D_{a} V_{b}\right\}$.

In fact, if we have coordinates $\left\{x^{i}\right\}$ on $M$ and we write $Y=\left\{y_{a}^{i}\right\}$, where $Y_{a}=y_{a}^{i} \frac{\partial}{\partial x^{i}}$ (so that $\left\{x^{i}, y_{a}^{j}\right\}$ are local coordinates for $F(M)$ ), then we can write $D_{a}$ as the vector field

$$
D_{a}=y_{a}^{i}\left(\frac{\partial}{\partial x^{i}}-\Gamma_{i j}^{k}(x) y_{b}^{j} \frac{\partial}{\partial y_{b}^{k}}\right) .
$$

The $D_{a}$ give us the vectors horizontal to the fibers of the frame bundle (we use the connection $\Gamma$ on the tangent bundle).

In addition, there are also the vector fields

$$
\nabla_{b}^{a}=y_{b}^{i} \frac{\partial}{\partial y_{a}^{i}}
$$

corresponding to transforming the $a$-axis into the $b$-axis. The $\nabla_{b}^{a}$ form a basis for the vectors tangent to the fibers of the frame bundle. Together, the $D_{a}$ and $\nabla_{b}^{a}$ give us a basis for the tangent vectors for the whole frame bundle.

Now, we wish to work with orthonormal frames only. This means, we want to consider the orthonormal frame bundle $O F(M)$. These are the frames $\left\{Y_{a}\right\}$ which satisfy $g\left(Y_{a}, Y_{b}\right)=I_{a b}$, where $I_{a b}$ is

$$
I_{a b}= \begin{cases}1 & \text { if } a=b \\ 0 & \text { if } a \neq b\end{cases}
$$

By compatibility of the Levi-Civita connection, we have that $D_{a} g_{b c}=\{0\}$, so that the vectors $D_{a}$ live on $O F(M)$. The $\nabla_{b}^{a}$ do not. However, the vectors, representing infinitesimal rotation, defined by 


$$
\delta_{a b}=g_{a c} \nabla_{b}^{c}-g_{b c} \nabla_{a}^{c},
$$

do satisfy $\delta_{a b} g_{c d}=\{0\}$. In fact, the $D_{a}$ and the $\delta_{a b}$ are a basis for the tangent vectors on the orthonormal frame bundle.

Under the Ricci flow, we also have to deal with the vector field $\frac{\partial}{\partial t}$. We decompose $\frac{\partial}{\partial t}$ into

$$
\frac{\partial}{\partial t}=D_{t}-R_{a b} g^{b c} \nabla_{c}^{a}
$$

where $D_{t}$ is the part which is tangent to the orthonormal frame bundle, and $-R_{a b} g^{b c} \nabla_{c}^{a}$ is orthogonal to it. (The equation $\frac{\partial}{\partial t} g_{a b}=-2 R_{a b}$ is used here). Again, as we wish to stay on the orthonormal frame bundle, $D_{t}$ is the vector field that we will work with.

We now work exclusively with orthonormal frames. All our indicies will be lowered and, as usual, repeated indices are summed.

Because, all our vector fields $D_{a}, \delta_{a b}$, and $D_{t}$ take $g_{c d}$ to zero, many computations are cleaner, particularly when we need to take the trace of an equation. With this setup, we now write the various evolution equations that we need. As a shorthand, we let

$$
\square=D_{t}-\Delta,
$$

where $\Delta=D_{e} D_{e}$. The curvature tensor $R_{a b c d}$ flows like

$$
\square R_{a b c d}=-2\left(B_{a b c d}-B_{a b d c}+B_{a c b d}-B_{a d b c}\right),
$$

where $B_{a b c d}=R_{a e b f} R_{c e d f}$. We trace this to obtain

$$
\square R_{a b}=2 R_{a c d b} R_{c d}
$$

and trace again to get

$$
\square R=2 R_{a b} R_{a b}=2|\mathrm{Rc}|^{2} .
$$

Our commutation equations are

$$
\begin{aligned}
D_{t} D_{a}-D_{a} D_{t} & =R_{a b} D_{b}+D_{b} R_{a c} \delta_{b c}, \\
D_{a} D_{b}-D_{b} D_{a} & =\frac{1}{2} R_{a b c d} \delta_{d c}, \\
\Delta D_{a}-D_{a} \Delta & =R_{a b} D_{b}+D_{b} R_{a c} \delta_{b c}-R_{a b c d} D_{b} \delta_{d c}, \\
\square D_{a}-D_{a} \square & =R_{a b c d} D_{b} \delta_{d c} .
\end{aligned}
$$




\section{Proof of the Theorem.}

We now prove our main theorem. Using orthonormal frames, we first rewrite our theorem as:

Theorem 7.1. Let $\frac{\partial}{\partial t} g=-2$ Rc. Let $A$ be a closed 2-form solving

$$
\square_{d} A=\left(D_{t}-\Delta_{d}\right) A=R_{a w} A_{w b}+A_{a w} R_{w b},
$$

and $h$ a symmetric two-tensor solving

$$
\square_{L} h=\left(D_{t}-\Delta_{L}\right) h=D_{a} D_{b}|A|^{2}+R_{a w} h_{w b}+R_{w b} h_{a w}
$$

Then, the inequality

$$
\Phi=R_{a b c d} U_{a b} U_{d c}+2 W_{c} D_{c} A_{a b} U_{b a}+\left(A_{a w} A_{b w}+h_{a b}\right) W_{a} W_{b} \geq 0
$$

is preserved.

Remark 7.2. We note that for two-forms $T_{a b}$, we have that the Hodge Laplacian looks like:

$$
\Delta_{d} T_{a b}=\Delta T_{a b}+2 R_{c a b d} T_{c d}-R_{a w} T_{w b}-R_{w b} T_{a w},
$$

so that

$$
\square_{d} T_{a b}=\square T_{a b}-2 R_{c a b d} T_{c d}+R_{a w} T_{w b}+R_{w b} T_{a w},
$$

and thus our condition that

$$
\square_{d} A=\left(D_{t}-\Delta_{d}\right) A=R_{a w} A_{w b}+A_{a w} R_{w b}
$$

is equivalent to

$$
\square A_{a b}=2 R_{c a b d} A_{c d} .
$$

Also, we have, by definition,

$$
\Delta_{L} T_{a b}=\Delta T_{a b}+2 R_{c a b d} T_{c d}-R_{a w} T_{w b}-R_{w b} T_{a w},
$$

giving

$$
\square_{L} T_{a b}=\square T_{a b}-2 R_{c a b d} T_{c d}+R_{a w} T_{w b}+R_{w b} T_{a w} .
$$

So that,

$$
\square_{L} h=\left(D_{t}-\Delta_{L}\right) h=D_{a} D_{b}|A|^{2}+R_{a w} h_{w b}+R_{w b} h_{a w}
$$

is the same as

$$
\square h_{a b}=D_{a} D_{b}|A|^{2}+2 R_{c a b d} h_{c d} .
$$


Remark 7.3. It is interesting to note that though our LYH quantity is derived from space-time considerations, the proof that follows is more reminiscent of Hamilton's original proof of the LYH estimate.

Proof. We will use the maximum principle on the quantity $\Phi$. The bulk of the work here is to compute the evolution equation of $\Phi$ and to put it into a form where we can apply the maximum principle. So, we compute

$$
\begin{aligned}
\square \Phi=( & \left.\square R_{a b c d}\right) U_{a b} U_{d c}+R_{a b c d}\left(\square U_{a b}\right) U_{d c} \\
& -4 D_{w} R_{a b c d}\left(D_{w} U_{a b}\right) U_{d c}-2 R_{a b c d} D_{w} U_{a b} D_{w} U_{d c} \\
& +2 \square W_{c}\left(D_{c} A_{a b}\right) U_{b a}+2 W_{c}\left(\square D_{c} A_{a b}\right) U_{b a}+2 W_{c}\left(D_{c} A_{a b}\right) \square U_{b a} \\
& -4 D_{w} W_{c}\left(D_{w} D_{c} A_{a b}\right) U_{b a}-4 D_{w} W_{c}\left(D_{c} A_{a b}\right) D_{w} U_{b a} \\
& -4 W_{c}\left(D_{w} D_{c} A_{a b}\right) \square U_{b a}+2\left(\square A_{a w}\right) A_{b w} W_{a} W_{b}+2 A_{a w} A_{b w}\left(\square W_{a}\right) W_{b} \\
& -2\left(D_{z} A_{a w}\right)\left(D_{z} A_{b w}\right) W_{a} W_{b}-2 A_{a w} A_{b w}\left(D_{z} W_{a}\right)\left(D_{z} W_{b}\right) \\
& -4\left(D_{z} A_{a w}\right) A_{b w}\left(D_{z} W_{a}\right) W_{b}-4\left(D_{z} A_{a w}\right) A_{b w} W_{a}\left(D_{z} W_{b}\right) \\
& +\left(\square h_{a b}\right) W_{a} W_{b}+2 h_{a b}\left(\square W_{a}\right) W_{b} \\
& -4\left(D_{w} h_{a b}\right)\left(D_{w} W_{a}\right) W_{b}-2 h_{a b}\left(D_{w} W_{a}\right)\left(D_{w} W_{b}\right) .
\end{aligned}
$$

And then, regroup terms to get

$$
\begin{aligned}
\square \Phi=( & \left.\square R_{a b c d}\right) U_{a b} U_{d c} \\
& +2 W_{c}\left(\square D_{c} A_{a b}\right) U_{b a}+2\left(\square A_{a w}\right) A_{b w} W_{a} W_{b} \\
& -2\left(D_{z} A_{a w}\right)\left(D_{z} A_{b w}\right) W_{a} W_{b}+\left(\square h_{a b}\right) W_{a} W_{b}+R_{a b c d}\left(\square U_{a b}\right) U_{d c} \\
& -4 D_{w} R_{a b c d}\left(D_{w} U_{a b}\right) U_{d c}-2 R_{a b c d} D_{w} U_{a b} D_{w} U_{d c} \\
& +2 \square W_{c}\left(D_{c} A_{a b}\right) U_{b a}+2 W_{c}\left(D_{c} A_{a b}\right) \square U_{b a} \\
& -4 D_{w} W_{c}\left(D_{w} D_{c} A_{a b}\right) U_{b a}-4 D_{w} W_{c}\left(D_{c} A_{a b}\right) D_{w} U_{b a} \\
& -4 W_{c}\left(D_{w} D_{c} A_{a b}\right) \square U_{b a}+2 A_{a w} A_{b w}\left(\square W_{a}\right) W_{b}+2 h_{a b}\left(\square W_{a}\right) W_{b} \\
& -2 A_{a w} A_{b w}\left(D_{z} W_{a}\right)\left(D_{z} W_{b}\right)-4\left(D_{z} A_{a w}\right) A_{b w}\left(D_{z} W_{a}\right) W_{b} \\
& -4\left(D_{z} A_{a w}\right) A_{b w} W_{a}\left(D_{z} W_{b}\right) \\
& -4\left(D_{w} h_{a b}\right)\left(D_{w} W_{a}\right) W_{b}-2 h_{a b}\left(D_{w} W_{a}\right)\left(D_{w} W_{b}\right),
\end{aligned}
$$

where the first five lines don't involve any derivatives on $W_{a}$ or on $U_{a b}$, and the others do. Now, using our various flow equations, we expand the first five lines explicitly, concluding that they are equal to

$$
\begin{aligned}
& -4 R_{a m b n} R_{c m d n} U_{a b} U_{d c}-4 R_{a m c n} R_{b m d n} U_{a b} U_{d c} \\
& +4 W_{c}\left(D_{c} R_{m a b n}\right) A_{m n} U_{b a}+4 W_{c} R_{m a b n}\left(D_{c} A_{m n}\right) U_{b a}+8 R_{d c w a} D_{d} A_{w b} U_{b a} \\
& +4 R_{m a w n} A_{m n} A_{b w} W_{a} W_{b}-2\left(D_{z} A_{a w}\right)\left(D_{z} A_{b w}\right) W_{a} W_{b} \\
& +2\left(D_{a} D_{b} A_{c d}\right) A_{c d} W_{a} W_{b}+2\left(D_{a} A_{c d}\right)\left(D_{b} A_{c d}\right) W_{a} W_{b}+2 R_{m a b n} h_{m n} W_{a} W_{b}
\end{aligned}
$$


To deal with the terms involving the derivatives of $W_{a}$ or on $U_{a b}$, we first prescribe

$$
D_{a} W_{b}=0, \square U_{a b}=0, \square W_{a}=0 .
$$

As with Hamilton's LYH quantity, ours does not involve any derivatives of $W$ or $U$, so we are allowed to prescribe values for these derivatives. This kills all of these derivative terms except for

$$
-4\left(D_{w} R_{a b c d}\right)\left(D_{w} U_{a b}\right) U_{d c}-2 R_{a b c d}\left(D_{w} U_{a b}\right)\left(D_{w} U_{d c}\right)-4 W_{c}\left(D_{w} D_{c} A_{a b}\right) D_{w} U_{b a}
$$

So that at this point, we have

$$
\begin{aligned}
\square \Phi=- & 4 R_{a m b n} R_{c m d n} U_{a b} U_{d c}-4 R_{a m c n} R_{b m d n} U_{a b} U_{d c} \\
& +4 W_{c}\left(D_{c} R_{m a b n}\right) A_{m n} U_{b a}+4 W_{c} R_{m a b n}\left(D_{c} A_{m n}\right) U_{b a} \\
& +8 R_{d c w a} D_{d} A_{w b} U_{b a}+4 R_{m a w n} A_{m n} A_{b w} W_{a} W_{b} \\
& -2\left(D_{z} A_{a w}\right)\left(D_{z} A_{b w}\right) W_{a} W_{b}+2\left(D_{a} D_{b} A_{c d}\right) A_{c d} W_{a} W_{b} \\
& +2\left(D_{a} A_{c d}\right)\left(D_{b} A_{c d}\right) W_{a} W_{b}+2 R_{m a b n} h_{m n} W_{a} W_{b} \\
& -4\left(D_{w} R_{a b c d}\right)\left(D_{w} U_{a b}\right) U_{d c}-2 R_{a b c d}\left(D_{w} U_{a b}\right)\left(D_{w} U_{d c}\right) \\
& -4 W_{c}\left(D_{w} D_{c} A_{a b}\right) D_{w} U_{b a} .
\end{aligned}
$$

We have not yet prescribed values for $D_{w} U_{a b}$. To further simplify our expression, we let

$$
D_{w} U_{a b}=\frac{1}{2}\left(W_{a} A_{b w}-W_{b} A_{a w}\right) .
$$

With this choice, we conclude that the evolution equation of the LYH quantity $\Phi$ is

$$
\begin{aligned}
\square \Phi=- & 4 R_{a m b n} R_{c m d n} U_{a b} U_{d c}-4 R_{a m c n} R_{b m d n} U_{a b} U_{d c} \\
& +4 W_{c} R_{m a b n}\left(D_{c} A_{m n}\right) U_{b a}+8 W_{c} R_{d c w a}\left(D_{d} A_{w b}\right) U_{b a} \\
& -2\left(D_{z} A_{a w}\right)\left(D_{z} A_{b w}\right) W_{a} W_{b} \\
& +2\left(D_{a} A_{c d}\right)\left(D_{b} A_{c d}\right) W_{a} W_{b}+2 R_{m a b n} h_{m n} W_{a} W_{b} \\
& +2 W_{a} W_{d} R_{a b c d} A_{b w} A_{c w} .
\end{aligned}
$$

After careful examination of the terms here, we discover that the evolution equation of $\Phi$ may be written as the sum of four terms, plus a square:

$$
\begin{aligned}
\square \Phi= & 4 R_{a m c n} R_{b m d n} U_{a b} U_{c d}+8 W_{c} R_{d c w a}\left(D_{d} A_{w b}\right) U_{b a} \\
& -2\left(D_{d} A_{a c}\right)\left(D_{c} A_{b d}\right) W_{a} W_{b} \\
& +2 R_{m a b n}\left(h_{m n}+A_{m w} A_{n w}\right) W_{a} W_{b} \\
& +\left[R_{a b c d} U_{c d}+W_{c} D_{c} A_{a b}\right]\left[R_{a b u v} U_{u v}+W_{u} D_{u} A_{a b}\right] .
\end{aligned}
$$


However, we also have that the sum of the first four terms are non-negative when $\Phi$ is non-negative. To see this, we "diagonalize" $\Phi$ by writing

$$
\begin{aligned}
\Phi & =R_{a b c d} U_{a b} U_{d c}+2 W_{c}\left(D_{c} A_{a b}\right) U_{b a}+\left(A_{a w} A_{b w}+h_{a b}\right) W_{a} W_{b} \\
& =\sum\left(X_{a}^{N} W_{a}+Y_{a b}^{N} U_{a b}\right)^{2} \\
& =\sum X_{a}^{N} X_{c}^{N} W_{a} W_{c}+X_{a}^{N} Y_{c d}^{N} W_{a} U_{c d}+X_{c}^{N} Y_{a b}^{N} W_{c} U_{a b}+Y_{a b}^{N} Y_{c d}^{N} U_{a b} U_{c d}
\end{aligned}
$$

so that $\Phi$ is a sum of squares. This forces

$R_{a b c d}=\sum_{N} Y_{a b}^{N} Y_{d c}^{N}, D_{c} A_{a b}=\sum_{N} Y_{a b}^{N} X_{c}^{N}$, and $\left(A_{a w} A_{b w}+h_{a b}\right)=\sum_{N} X_{a}^{N} X_{b}^{N}$,

where, the $X_{a}^{N}$ 's are one-forms, and the $Y_{a b}^{N}$ 's are two-forms for each $N$. This makes

$$
\begin{aligned}
& 4 R_{a m c n} R_{b m d n} U_{a b} U_{c d}+8 W_{c} R_{d c w a}\left(D_{d} A_{w b}\right) U_{b a} \\
& -2\left(D_{d} A_{a c}\right)\left(D_{c} A_{b d}\right) W_{a} W_{b}+2 R_{m a b n}\left(h_{m n}+A_{m w} A_{n w}\right) W_{a} W_{b} \\
& =\sum_{M, N}\left(Y_{a c}^{M} X_{c}^{N} W_{a}-Y_{a c}^{N} X_{c}^{M} W_{a}-2 Y_{a c}^{M} Y_{b c}^{N} U_{a b}\right)^{2}
\end{aligned}
$$

so that,

$$
\begin{aligned}
\square \Phi= & \sum_{M, N}\left(Y_{a c}^{M} X_{c}^{N} W_{a}-Y_{a c}^{N} X_{c}^{M} W_{a}-2 Y_{a c}^{M} Y_{b c}^{N} U_{a b}\right)^{2} \\
& +\left[R_{a b c d} U_{c d}+W_{c} D_{c} A_{a b}\right]\left[R_{a b u v} U_{u v}+W_{u} D_{u} A_{a b}\right] \\
& \geq 0 .
\end{aligned}
$$

Now, the maximum principle is applied and we are done.

\section{Acknowledgments.}

I would like to thank Bennett Chow and Shing-Tung Yau for their indispensable advice, support, and encouragement. Also, thanks to Lei $\mathrm{Ni}$ and Jiaping Wang for their encouragement and mathematical discussions.

\section{References.}

[1] H.-D. Cao, On Harnack's inequalities for the Kähler-Ricci flow, Invent. Math. 109 (1992), 247-263. 
[2] B. Chow, On Harnack's inequality and entropy for the Gaussian curvature flow, Comm. Pure Appl. Math. 44 (1996), 249-264.

[3] B. Chow and S.-C. Chu, A geometric interpretation of Hamilton's Harnack inequality for the Ricci flow, Math. Res. Lett. 2 (1995), 701-708.

[4] B. Chow and S.-C. Chu, A geometric approach to the linear trace Harnack inequality for the Ricci flow, Math. Res. Lett. 3 (1996), 549-568.

[5] B. Chow and D. Knopf, New Li-Yau-Hamilton Inequalities for the Ricci flow via the space-time approach, J. Differential Geom. 60 (2002), 1-54.

[6] R. S. Hamilton, Four-manifolds with positive curvature operator, J. Differential Geom. 24 (1986), 153-179.

[7] R. S. Hamilton, The Ricci flow on surfaces, Contemporary Mathematics 71 (1988), 237-261.

[8] R. S. Hamilton, The Harnack Estimate for the Ricci Flow, J. Differential Geom. 37 (1993), 225-243.

[9] R. S. Hamilton, Eternal solutions to the Ricci flow, J. Differential Geom. 38 (1993), 1-11.

[10] R. S. Hamilton, The formation of singularities in the Ricci flow, Surveys in Diff. Geom. International Press 2 (1995), 7-136.

[11] R. S. Hamilton, Harnack estimate for the mean curvature flow, J. Differential Geom. 41 (1995), 215-226.

[12] R. S. Hamilton and S.-T. Yau, The Harnack estimate for the Ricci flow on a surface - revisited, Asian J. Math 1 (1997), 418-421.

[13] T. Ivey, Ricci solitons on compact 3-manifolds, Differential Geom. Appl. 3 (1993), 301-307.

[14] G. Perelman, The entropy formula for the Ricci flow and its geometric applications, arXiv:math.DG/0211159v1, (2002). 